\title{
Diagnostic Utility of Immunohistochemical Markers in Prostate Cancer
}

\author{
Dr. O.D.E.Anand ${ }^{1}$,Dr. P. Satyanarayana $\mathrm{Rao}^{2}$,Dr. Ch. Kishore Kumar ${ }^{3}$, \\ Dr. R. Vijaya Bhaskar ${ }^{4}$ \\ ${ }^{I}$ (Assistant Professor, Department of Pathology, Konaseema Institute of Medical Sciences \& RF, \\ Amalapuram, Andhra Pradesh, India) \\ ${ }_{2}^{2}$ (Associate Professor, Department of Pathology, Rangaraya Medical College, Kakinada, \\ Andhra Pradesh, India) \\ ${ }^{31}$ Assistant Professor, Department of Pathology, Rangaraya Medical College, Kakinada, \\ Andhra Pradesh, India) \\ ${ }^{4}$ (Professor \& Head, Department of Pathology, Rangaraya Medical College, Kakinada, \\ Andhra Pradesh, India)
}

\begin{abstract}
Introduction: Prostate cancer is a multifaceted disease comprises distinct biological subtypes with varied spectrum of clinical, pathologic and molecular features with different prognostic and therapeutic implications. Over the last few decades there have been outstanding advances in prostate cancer management leading to earlier detection of disease. Prostatic cancer diagnosis is based on a combination of architectural, cytological and ancillary features.

Immunohistochemistry $(\mathrm{IHC})$ is a valuable adjunctive in diagnosis of minute foci of prostatic carcinoma and to differentiate it from benign mimickers \& precursor lesions. Prostate cancer is now the 5th most common cancer in the world (in terms of number of new cases), and 2nd most common cancer in men.

Aims of study :1) To assess the diagnostic utility of IHC markers in morphologically ambiguous prostate carcinomas 2) To assess the efficacy of P63 over HMWCK 3) To know the level of diagnostic error in reporting of Prostatic lesions on routine $H \& E$.

Materials \&Methods : The Present study is a prospective study carried for a period of two years from June 2011 to May 2013 in Department of Pathology, Rangaraya medical college / Government general hospital, Kakinada. We received 310 cases of prostate biopsies in our department in the two year period. Out of the 310 cases, 40 cases were reported as suspicious of malignancy on $H \& E$ and are subjected for IHC.

Results \&Analysis : 40 suspicious cases were subjected for IHC. Out of 40 cases, in only 20 cases (50\%) there is disparity between diagnosis on H\&E and diagnosis on IHC. In 20 cases, 14 cases (35\%) were underdiagnosed as benign or pre-malignant or suspicious of malignancy. These got upgraded to HGPIN or carcinoma or carcinoma with foci of HGPIN after IHC study. Remaining 6 cases (15\%) are downgraded from pre-malignant lesions to benign lesions.

Discussion : Our results are almost correlated with other studies.

Conclusion : 1.Even though diagnosis of prostate cancer is mainly based on architectural pattern, cytological and ancillary features, Immunohistochemistry acts as a most valuable adjunctive, which significantly increases the diagnostic accuracy in prostatic carcinoma.

2.Among basal cell markers, $p 63$ has more sensitivity and specificity than HMWCK.

3.No single marker is having $100 \%$ sensitivity or specificity in diagnosis of prostatic carcinoma. Hence combination of a Basal cell marker (p63) with AMACR is more informative in the diagnosis of prostatic carcinoma.
\end{abstract}

Keywords: Prostate carcinoma, Alpha methyl Co-A racemase (AMACR), Basal cell markers - HMWCK, P63.

\section{Introduction}

Prostate cancer is a multifaceted disease comprises of distinct biological subtypes with diverse natural history, presenting a varied spectrum of clinical, pathologic and molecular features with different prognostic and therapeutic implications. Over the last few decades there have been outstanding advances in prostate cancer management leading to earlier detection of disease.

Prostatic cancer diagnosis is based on a combination of architectural, cytological and ancillary features. Immunohistochemistry (IHC) is a valuable adjunctive in diagnosis of minute foci of prostatic carcinoma and to differentiate it from benign mimickers \& precursor lesions. Prostate cancer is now the 5 th most common cancer in the world (in terms of number of new cases), and 2nd most common cancer in men. 
Three IHC markers have been proved useful in the diagnosis ambiguous cases of Prostate Carcinoma. (Flow chart 1):

- $\alpha$-methyl-acyl-coenzyme A racemase (AMACR), a positive diagnostic tissue biomarker of prostate cancer,

- Lack of staining with basal cell markers (34ßE12-HMWCK \& p63) supports the diagnosis of carcinoma in ambiguous cases.

\section{Materials \& Methods}

The present study is a prospective study carried for a period of two years from June 2011 to May 2013 in Department of Pathology, Rangaraya medical college / Government general hospital, Kakinada. We received 310 cases of prostate biopsies in our department in the two year period. Out of the 310 cases, 226 cases were benign, 44 cases were frank malignancies and 40 are suspicious / premalignant cases. All the blocks and $H \& E$ stained slides of 40 suspicious cases were collected. For the TURP specimens, suspected chips were marked on the slide and were separated from the block and re-embedded, are subjected to IHC. For open prostatectomy specimens, one block with suspicious foci is selected and subjected to IHC. For core needle biopsies entire core is subjected to IHC.The cases with suspicious foci were subjected to immunohistochemistry (IHC) using HMWCK (34bE12), P63 and AMACR (p504S) markers. To act as controls 11 cases of proven carcinomas of various grades and 5 benign cases were taken.The 40 suspicious cases consists 14 open prostatectomies, 1 core needle biopsy and 25 were TURP chips. The age group of patients in present study is ranged from 50 to $95 \mathrm{yrs}$.

IHC staining of biopsies were performed in Department of Pathology, GGH, Kakinada and these were divided into various categories like BPH, Basal cell hyperplasia, Cribriform hyperplasia, AAH, ASAP, HGPIN, Carcinoma.

\section{Results}

Out of 40 cases selected to IHC, in only 20 cases $(50 \%)$ there is disparity between diagnosis on $\mathrm{H} \& \mathrm{E}($ Table 1\&2) and diagnosis on IHC. In 20 cases, 14 cases (35\%) were under-diagnosed as benign or premalignant or suspicious of malignancy. These got upgraded to HGPIN or carcinoma or carcinoma with foci of HGPIN. Remaining 6 cases $(15 \%)$ are downgraded from pre-malignant lesions to benign lesions.IHC was performed on the 40 suspicious cases using two basal cell markers HMWCK \& P63 and a positive marker for carcinoma AMACR. After IHC staining results interpretated as circumferential \& strong diffuse positivity with AMACR \& negative stains with HMWCK \& p63 - labelled as adenocarcinoma.Negative/ weak non circumferential AMACR stains \& positive HMWCK \&p63 in basal cells considered as adenosis/Atrophy.Positive staining with HMWCK \& p63 in the luminal cells labelled as basal cell hyperplasia.

Suspicious foci with large glands exhibiting positive staining with both AMACR \& HMWCK, P63 taken as HGPIN. Under these guidelines all 40cases subjected to IHC were interpreted Out of the 40 suspicious cases 14 cases (35\%) are under-diagnosed (FIGURE 1 \& 2) in which 9 (22.5\%) cases were carcinomas, 2 cases were carcinoma with foci of HGPIN (5\%) and 3 cases (7.5\%) were HGPIN. Out of the 6 cases (15\%) which got downgraded on IHC (FIGURE 3), 3 cases (7.5\%) were basal cell hyperplasias, 2 cases (5\%) were AAH and 1 case $(2.5 \%)$ was BPH. Finally out of 40 suspicious cases, 26 cases $(65 \%)$ were benign and 14 cases $(35 \%)$ were diagnosed as malignant or premalignant lesions on application of IHC markers (Table $3 \& 4$ ). Out of the 40 suspicious cases, in 31 cases there is good correlation in staining of basal cells by P63 and HMWCK. In rest of the 9 cases, show superior basal cell layer staining by p63 when compared to HMWCK.

\section{Discussion}

In 2001, Jiang et al examined AMACR expression in 137 prostatic carcinomas and 70 benign prostate cases by IHC using rabbit monoclonal antibody of AMACR. All 137 cases (100\%) showed strong positivity irrespective of their Gleasons grade. In addition $88 \%$ of benign lesions show complete negativity for AMACR, with focal weak positivity in $12 \%$ of cases ${ }^{1}$. This is correlated with our study, where there is $100 \%$ positivity of Prostatic cancer controls (11/11) and $20 \%$ of benign controls (1/5) show focal weak positivity with AMACR.

According to Zhong Jiang et al, using AMACR as a positive marker alone might be misleading because weak expression of AMACR might be seen in benign glands, and expression of AMACR is seen in HGPIN and AAH. To avoid such errors, in our study along with AMACR we used basal cell markers also, which will enhance the diagnostic accuracy. ${ }^{1}$

Zhou et al demonstrated that, of 115 prostate biopsies diagnosed as atypical lesions by an expert pathologist, $34(30 \%)$ were changed to a final diagnosis of cancer based on a positive AMACR immunostain Molinie et al were able to resolve $89 \%$ of 104 "ASAP" cases in needle biopsies using p63/AMACR antibody cocktail compared with only $53 \%$ with CK $5 / 6 .^{2}$

Kunju et al were able to resolve 27 (93\%) of 29 atypical biopsies after immunostaining with AMACR and basal cell marker. ${ }^{3}$ In our study diagnosis of 40 suspicious cases (100\%) are resolved using two basal cell 
markers (P63 \& HMWCK) and AMACR (Table 5). M.H. Weinstein et al proved that p63 is more superior in demonstrating Prostatic Basal Cells when compared to $\mathrm{HMWCK}^{4}$ which correlated with our study. Our results reveal high expression of p63 (40 of 40,100\%) in normal basal cells and confirm the superiority over HMWCK (31 of $40,77.5 \%$ ), with an improvement in specificity.Beach et al found $82 \%$ AMACR expression in prostate cancer whatever may be the degree of differentiation i.e. Gleason score or morphological types. In our study controls we got $100 \%$ positivity in all grades and variants of prostatic carcinoma. In one case of foamy cell prostatic carcinoma there is focal expression of AMACR, but it is dark and circumferential staining, so interpreted as positive. In one case of high grade prostatic carcinoma, there is focal but dark staining of cytoplasm of the cancer cells and hence interpreted as positive. These results are also in agreement with the study of Jiang et al, who found numerous benign hypertrophic hyperplastic nodules negative for AMACR staining, indicating that cancer development is restricted to a subset of hypertrophic lesions which are premalignant or malignant, which may be identified by the enhanced expression of AMACR. ${ }^{5}$

Yang and colleagues found that AMACR was focally expressed in $10 \%$ of cases and diffusely positive in only $7.5 \%$ of cases of $\mathrm{AAH}^{7}$ In our study 1 out of $8 \mathrm{AAH}$ cases (12.5\% of AAH cases) showed focal expression of AMACR. In our study out of 40 suspicious cases, 26 were benign on IHC, of which 5 cases (12.5\%) showed weak, focal, discontinuous positivity which were interpreted as negative.In 2003, Kunju et al directly compared the two antibodies and found that $68 \%$ of benign glands showed weak expression of AMACR with polyclonal antibody compared to 7\% using the monoclonal antibody P504S (AMACR).This has correlated with our study as we used rabbit monoclonal antibody which is more sensitive of all as said by Kunju et al. ${ }^{8}$

\section{Summary}

The basic architectural and cytological features can help only to some extent, the valuable adjunctive in these cases is IHC.

\section{The two broad indications for use of immunohistochemistry in prostatic pathology:}

(1) In confirming prostate carcinoma and/or distinguishing it from its many benign mimics,

(2) In distinguishing prostate carcinoma from non-prostatic malignancies that secondarily involve the prostate.

Prostate-specific antigen (PSA) and prostatic acid phosphatase (PAP) traditionally have been used to confirm a prostatic tumor origin; however, they are not expressed uniformly in poorly differentiated Prostatic Carcinoma and might be negative in up to $27 \%$ and $19 \%$ of cases, respectively. They are mainly used to differentiate primary prostatic adenocarcinomas from metastatic adenocarcinomas. So, we conclude that in conjunction with morphology and clinical scenario, a combination of a basal cell marker (P63) and AMACR is of great value in combating the morphologically suspicious cases and thus significantly increasing the diagnostic accuracy in prostate cancer.

\section{Conclusion}

1. Even though diagnosis of prostate cancer is mainly based on architectural pattern, cytological and ancillary features, Immunohistochemistry acts as a most valuable adjunctive, which significantly increases the diagnostic accuracy in prostatic carcinoma.

2. Among basal cell markers, $\mathrm{p} 63$ has more sensitivity and specificity than HMWCK.

3. No single marker is having $100 \%$ sensitivity or specificity in diagnosis of prostatic carcinoma. Hence a combination of a Basal cell marker (p63) with AMACR is more informative in the diagnosis of prostatic carcinoma.

\section{References}

[1]. Using an AMACR (P504S)/34 Beta E12/p63 Cocktail for the Detection of Small Focal Prostate Carcinoma in Needle Biopsy Specime Zhong Jiang, MD, Cuizhen Li, MD, PhD, Andrew Fischer, MD, Karen Dresser, and Bruce A. Woda, MD Am J ClinPathol. 2005;123(2):231-236.

[2]. Diagnostic utility of a p63/a-methyl-CoA racemas (p504s) cocktail in atypical foci in the prostate Vincent Molinie'1, Gae"1le Fromont2, Mathilde Sibony3, Annick Vieillefond4 Viorel Vassiliu5, Be'atrix CochandPriollet6, Jean M Herve'7, Thierry Lebret7 and Anne C Baglin1 Modern Pathology (2004) 17, 1180-1190\&2004 USCAP.

[3]. Prostate-Specific Antigen, High-Molecular-Weight Cytokeratin (Clone 34ßE12), and/or p6 An Optimal Immunohistochemical Panel to Distinguish Poorly Differentiated Prostate Adenocarcinoma From Urothelial Carcinoma Lakshmi P. Kunju, MD,1RohitMehra, MD,1 Matthew Snyder, MD,1 and Rajal B. Shah, MD1,2 Am J Clin Pathol2006;125:675-681

[4]. Diagnostic Utility of Immunohistochemical Staining for p63, a Sensitive Marker of Prostatic Basal Cells Michael H. Weinstein, M.D., Ph.D., Sabina Signoretti, M.D., Massimo Loda, M.D. Department of Pathology, Brigham and Women's Hospital (MHW, ML) and the Dana Farber CancerInstitute (ML, SS), Boston, Massachusetts Modern Pathology; Augusta15.12 (Dec 2002): 1302-8.

[5]. P504S ( Methylacyl CoA Racemase) : A Good Diagnostic Marker for Prostate Carcinoma Zhong Jiang, MD Advances in Anatomic Pathology: November 2004 - Volume 11 - Issue 6 - pp 316-321

[6]. Alpha-Methylacyl Coenzyme A Racemase as a Tissue Biomarker for Prostate Cancer. Mark A. Rubin, MD Ming Zhou, MD, PhD Saravana M. Dhanasekaran, PhD SooryanarayanaVarambally, PhD Terrence R. Barrette Martin G. Sanda, MD Kenneth J. Pienta, MDDebashisGhosh, PhD Arul M. Chinnaiyan, MD, PhD JAMA. 2002;287(13):1662-1670. doi:10.1001/jama.287.13.1662 
[7]. Application of Alpha-methylacyl co-A racemase immunohistochemistry: A help or hindrance in the diagnosis of prostate cancer ? Ximing J. Yang, M.D., Ph.D. Department of Pathology, Feinberg 7-334

[8]. Northwestern Memorial Hospital Northwestern University, Feinberg School of Medicine

[9]. Diagnostic utility of $\alpha$-methylacyl CoA racemase (P504S) \& HMWCK in morphologically difficult prostate cancer Kumaresan et al. DiagnPathol. 2010; 5: 83. Published online 2010 Dec 22

[10]. Prostate-Specific Antigen, Prostate Cancer Screening, and the Pathologist What Should Be Our Role? Brian R. Jackson, MD, MS Arch Pathol Lab Med-Vol 137, March 2013, 308-309

[11]. rosai and ackerman's surgical pathology 10th Edition By Juan Rosai, MD, Director, International Center for Oncologic, Pathology Consultations, Centro DiagnosticoItaliano (CDI), Milan, Italy.

[12]. world health organization classification of tumours international agency for research on cancer (iarc) pathology and genetics of tumours of the urinary system and male genital organs Edited by John N. Eble, Guido Sauter, Jonathan I. Epstein, Isabell A. Sesterhenn

Flow Chart 1: Protocol of IHC markers interpretation

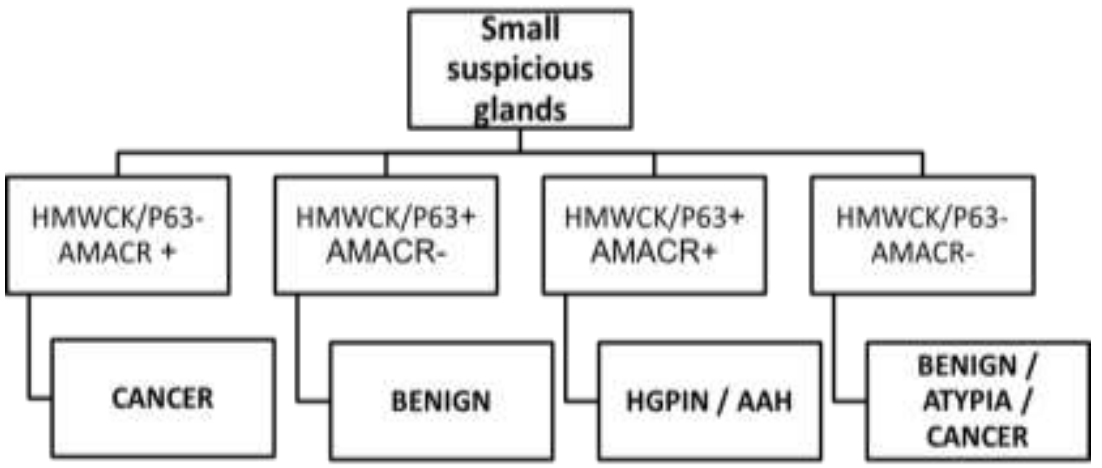

Table 1 : Diagnosis of total cases on H\&E staining

\begin{tabular}{|l|l|l|}
\hline Category & No. of cases & Percentage (\%) \\
\hline Benign & 226 & $72.9 \%$ \\
\hline $\begin{array}{l}\text { Suspicious \& pre- } \\
\text { malignant }\end{array}$ & 40 & $12.9 \%$ \\
\hline Malignant & 44 & $14.2 \%$ \\
\hline Total & $\mathbf{3 1 0}$ & $\mathbf{1 0 0 \%}$ \\
\hline
\end{tabular}

Table 2 : Diagnosis of suspicious cases on H\&E staining

\begin{tabular}{|l|l|l|}
\hline Type of lesion & No. of cases & Percentage \\
\hline Cribriform hyperplasia With ? PIN & & \\
& 2 & $5 \%$ \\
\hline ASAP & 5 & $12.5 \%$ \\
\hline ASAP with ? PIN & 6 & $15 \%$ \\
\hline AAH & 4 & $10 \%$ \\
\hline AAH with ?PIN & 7 & $17.5 \%$ \\
\hline AAH with metaplasia & 2 & $5 \%$ \\
\hline Basal cell hyperplasia & 2 & $5 \%$ \\
\hline PIN changes & 2 & $22.5 \%$ \\
\hline ?Carcinoma & 9 & $7.5 \%$ \\
\hline TOTAL & 3 & $100 \%$ \\
\hline & 40 & \\
\hline
\end{tabular}

Table 3 : Cases that are Underdiagnosed on $\mathrm{H} \& \mathrm{E}$

\begin{tabular}{|l|c|l|}
\hline Diagnosis on H\&E & No. of the cases & Diagnosis on IHC \\
\hline Basal cell hyperplasia & 1 & HGPIN \\
\hline Cribriform hyperplasia & 1 & HGPIN \\
\hline AAH & 1 & HGPIN \\
\hline ASAP with PIN & 2 & HGPIN with carcinoma \\
\hline ASAP & 1 & Carcinoma \\
\hline ASAP with PIN & 2 & Carcinoma \\
\hline AAH with PIN & 1 & Carcinoma \\
\hline ? Carcinoma & 3 & Carcinoma \\
\hline PIN & 2 & Carcinoma \\
\hline TOTAL & 14 & \\
\hline
\end{tabular}


Table 4 : Cases That Are Downgraded On IHC

\begin{tabular}{|l|l|l|}
\hline Diagnosis on H\&E & No. of the cases & Diagnosis on IHC \\
\hline AAH with PIN & 1 & BPH \\
\hline PIN changes & 3 & Basal cell hyperplasia \\
\hline PIN changes & 2 & AAH \\
\hline TOTAL & 6 & \\
\hline & & \\
\hline
\end{tabular}

Table 5 : Comparison With Other Studies

\begin{tabular}{|l|l|l|l|l|}
\hline Study & No. of cases & Markers used & $\begin{array}{l}\text { No. of cases } \\
\text { resolved }\end{array}$ & $\begin{array}{l}\text { Percentage } \\
(\%)\end{array}$ \\
\hline Browne et al & 123 & AMACR & 86 & $70 \%$ \\
\hline Molinie et al & 104 & $\begin{array}{l}\text { p63/AMACR } \\
\text { antibody cocktail }\end{array}$ & 93 & $89 \%$ \\
\hline Kunju et al & 29 & $\begin{array}{l}\text { AMACR and } \\
\text { basal cell markers }\end{array}$ & $\begin{array}{l}\text { AMACR \& } \\
\text { HMWCK }\end{array}$ & 49 \\
\hline Kumarsen et al
\end{tabular}

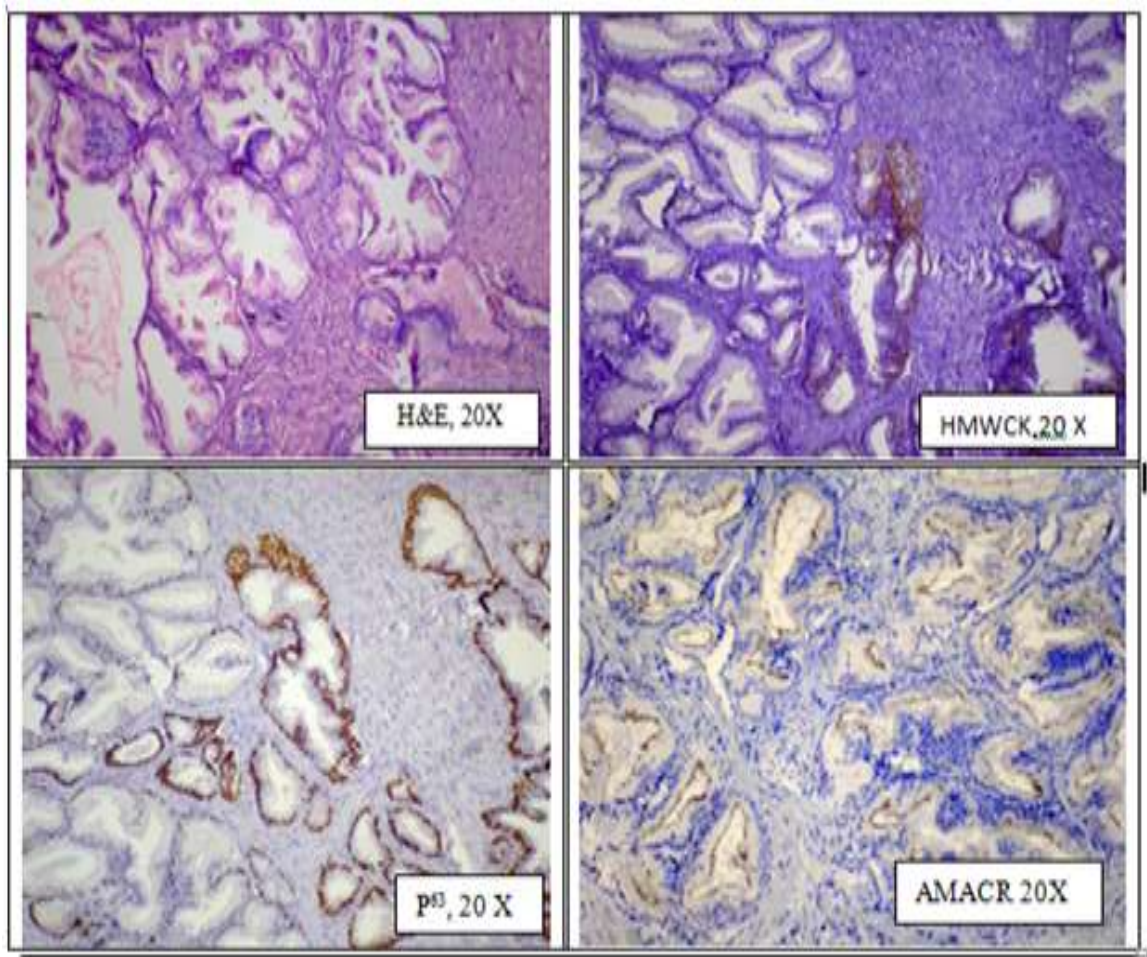

Figure 1: An under diagnosed case - Biopsy no.464/12

Diagnosis on H\&E:PIN changes;Final diagnosis on IHC:Carcinoma with HGPIN IHC : HMWCK \& P63 -ve (in crowded glands); AMACR +ve(in all glands) 

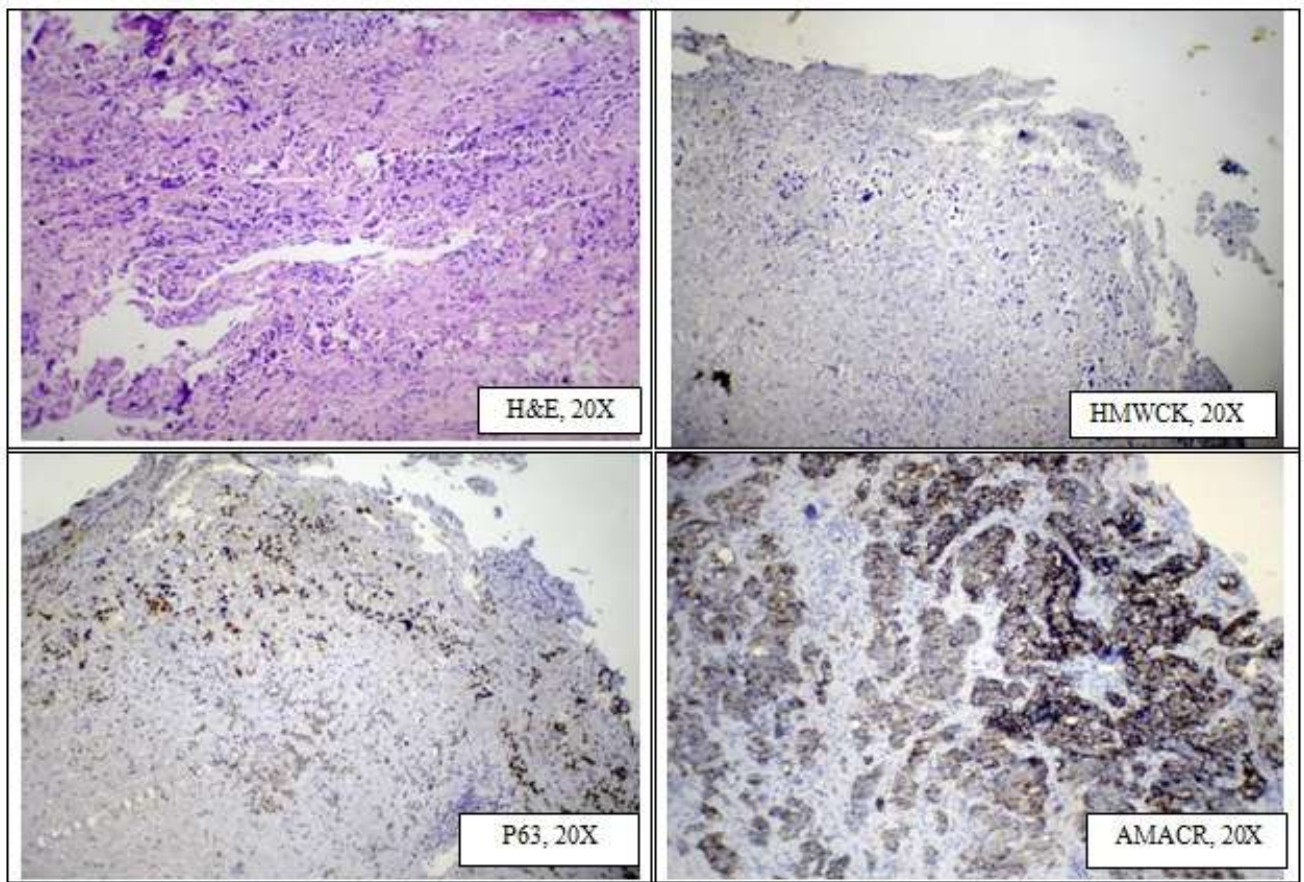

Figure 2 : An Under diagnosed case - Biopsy no.1982/13.

Diagnosis on H\&E : Suspicious of carcinoma; Final diagnosis on IHC : Carcinoma. IHC : HMWCK -ve; P63 Focally +ve; AMACR +ve diffusely

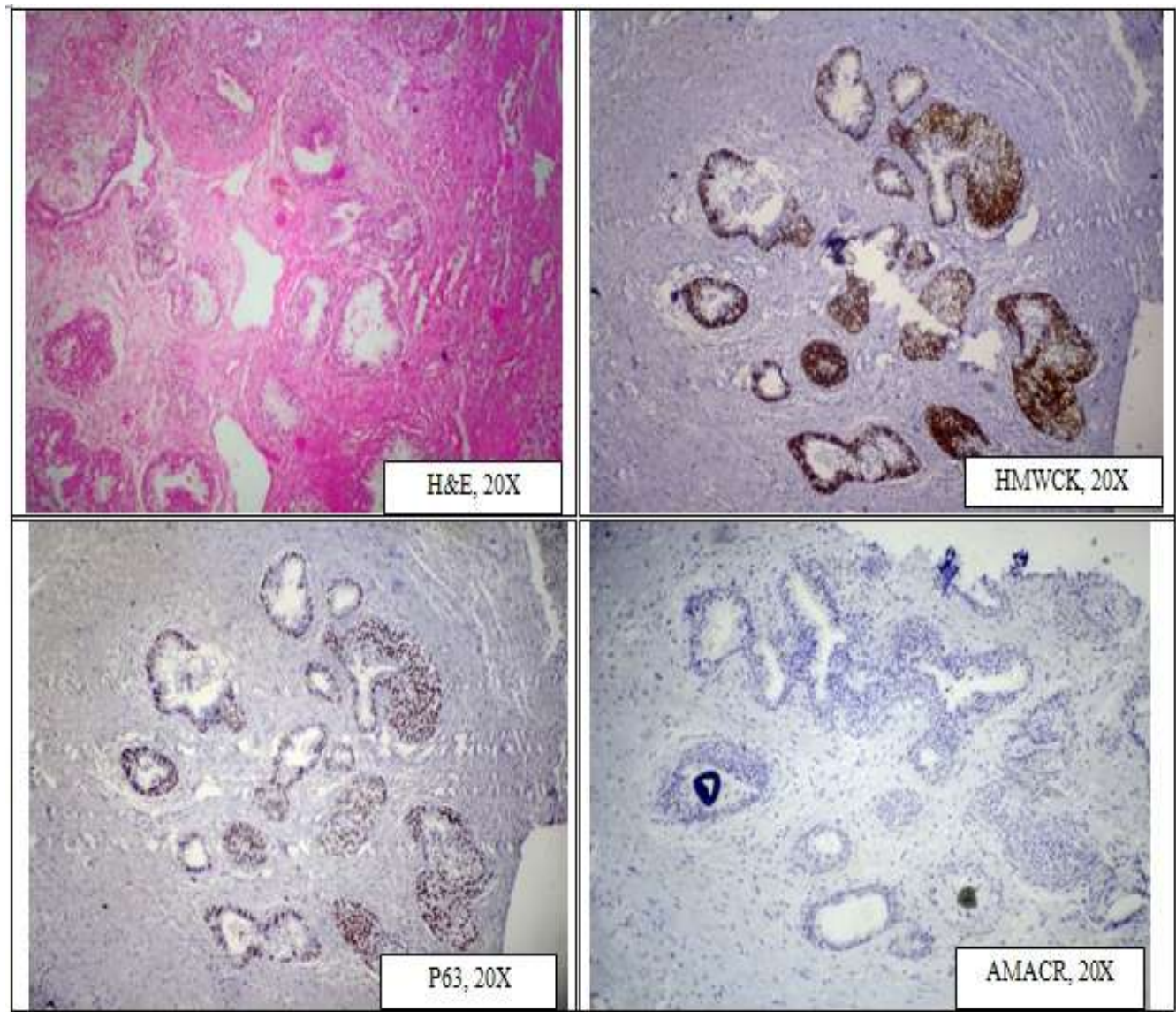

Figure 3 : A Downgraded Case - Biopsy 2639/11;

Diagnosis On H\&E : HGPIN ; Final Diagnosis Based On IHC : Basal Cell Hyperplasia IHC:HMWCK +ve; P 63 +ve; AMACR -ve. 
List Of Abbreviations Used

AAH Atypical Adenomatous Hyperplasia

AMACR Alpha Methylacyl Co-A Racemase

ASAP Atypical Small Acinar cell Proliferations

BPH Benign Prostatic Hyperplasia

DAB Diaminobenzidine tetrahydrochloride

HGPIN High Grade Prostatic Intraepithelial Neoplasia

HMWCK High Molecular Weight CytoKeratin (34ßE12)

H\&E Hematoxylin \& Eosin

IHC Immunohistochemistry

TURP Trans Urethral Resection of Prostate.

WHO World Health Organization 